\title{
Über die Ermittlung der räumlichen Anordnung im Molekül.
}

\author{
Von
}

E. Nickel in Berlin.

(Mit 1 Textfigur.)

Die Forschungen über die isomeren Kohlenstoffverbindungen haben bekanntlich in zablreichen Fällen darauf gefürt, dass gewissen Isomeren ein und dieselbe Strukturformel zugewiesen werden musste. Daraus ist je länger, je mehr die Notwendigkeit entstanden, die Strukturformeln im Sinne der räumlichen Lagerung weiter auszubilden, unt die hervorragendsten Forscher widmen sich jetzt - besonders bei uns in Deutschland - mit Vorliebe der Untersuchung der „struktur-identischen stereoisomeren" Verbindungen ${ }^{1}$ ).

Das Auftreten der Stereoisomeren beruht entweder auf einer gewissen Seitlichkeit des Moleküls durch das Auftreten einer charakteristischen Ebene im Atomgefüge, wie bei den Atomgruppen $C=C$ und $C=N$ und wie bei den Ringbildungen, oder auf der Gegenwart von sogen. asymmetrischen Kohlenstoffatomen. Die Anzahl der Isomeren letzterer Art, auf die sich die folgende Betrachtung unter Ausschluss der Seitlichkeitsisomerien beschränkt, steht zu der Zahl der asymmetrischen Kohlenstoffatome unter der Voraussetzung normaler Kohlenstoffketten in einer einfachen Beziehung. Das asymmetrische Kohlenstoffatom hat einen rechtsdrehenden oder linksdrehenden Charakter, für deren Bezeichnung im folgenden nach E. Fischer die Buchstaben $d$ und $l$ gebraucht werden sollen. Wenn wir diese beiden Charaktere $d$ und $l$ zu je $n$ kombinieren, so ist die Zahl der möglichen Kombinationen bekanntlich 2" (vergl. z. B. Hantzsch, Stereochemie 1893, S. 20). Wenn die Symbole $V^{\prime}, V^{\prime \prime}, V^{\prime \prime \prime}, \ldots \ldots$ die Anzahl der strukturidentischen stereoisomeren Verbindungen mit $1,2,3, \ldots \ldots$ asymmetrischen $C$-Atomen bedeuten, so ergiebt sich

$$
V^{\prime}=2, \quad V^{\prime \prime}=4, \quad V^{\prime \prime \prime}=8, \quad V^{\prime \prime \prime}=16, \ldots,
$$

1) Vgl. van't Hoff-Megerhoffer, Stereochemie 1892 und Victor Meger und Paul Jacobson, Organische Chemie 1893. 
Durch die Einführung weiterer Indices ergeben sich in jeder Gruppe für die Verbindungen selbst folgende Symbole:

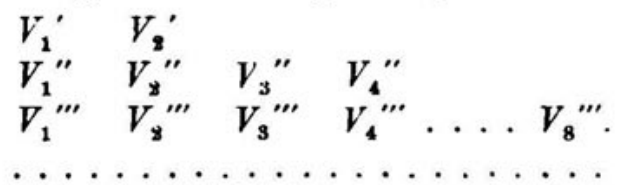

Die Verbindungen jeder Reihe sind unter sich strukturgleich, aber stereoisomer. Es sollen ausserdem die Verbindungen jeder Reihe mit denen der Nachbarreihe auf Grund ihrer Struktur entweder durch Anoder $\mathrm{Abbau}$ oder indirekt stammbaumartig zusammenhängen. Dabei soll die Kette der asymmetrischen $C$-Atome stets in derselben Richtung wachsen.

Bei der obigen Aufstellung sind natürlich die traubensäureartigen, die racemischen Formen; z. B. von dem Charakter $[d d]+[l l]$, ausgeschlossen. Es wird ferner vorausgesetzt, dass zunächst nicht eine besondere Beschaffenheit des Moleküls, die Symmetrie der Strukturformel, eine Verminderung der Anzahl der Stereoisomeren herbeiführt.

Man kann den Uberblick über den Charakter des räumlichen Gefäges, der Konfiguration, in den verschiedenen Fällen dadurch erleichtern, dass man das graphische Verfahren zu Hilfe nimmt.

Bezeichnen wir die Anzahl der asymmetrischen $C$-Atome von rechtsdrehendem Charakter mit $x$ und die von linksdrehendem Charakter mit $y$, so haben wir

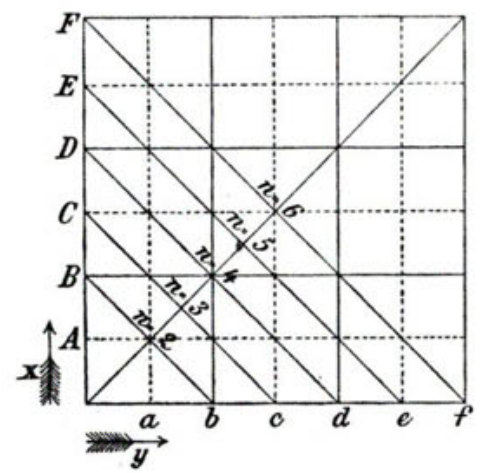

$$
\begin{aligned}
& n=x+y \\
& \frac{x}{n}+\frac{y}{n}=1 .
\end{aligned}
$$

Wählen wir $x$ und $y$ als Axenwerte und setzen wir der Reihe nach für $n$ die Zahl 1, 2, 3, 4 ..., so bekommen wir eine Schar von Parallelen, die unter einem Winkel von $45^{\circ}$ durch das graphische Feld hindurchlaufen. Ihre Schnittpunkte mit den Koordinaten für $x$ bezw. $y=$ $1,2,3,4 \ldots$ stellen verschiedene Charaktere, verschiedene Gattungen der Koufigurationen vor. Jeder Gattung gehören eine oder mehrere Spezies der Konfiguration an. Diese Zusammenfassung der Konfigurationsspezies zu Gattungen, zu der das graphische Verfahren führt, erleichtert, wie mir scheint, den Uberblick; denn schon für $n=4$ steigt die Anzahl der Kombinationen auf 16. 
Ein besonderes Interesse beanspruchen diejenigen Konfigurationen, deren Ort auf der Winkelhalbierenden des Feldes liegt. Wie sich aus der Tafel ergiebt, ist dieser Fall nur in Betracht zu ziehen, wenn $n$ eine gerade Zahl ist. Wenn er für die Konfigurationsbestimmung von Nutzen sein soll, so ist eine Vereinfachung des allgemeinen Falles notwendig. Die optische Inaktivität durch intramolekulare Kompensation ron Verbindungen der Gattung $\frac{n}{2} d \frac{n}{2} l$ wird mit Sicherheit nur eintreten bei Symmetrie der Gesamtformel. Wir müssen, vom allgemeinen Fall ausgehend, erst die Umwandlung der untersuchten Verbindung in eine solche mit symmetrischer Gesamtformel durchführen. Abkömmlinge dieser Art sollen kurz als struktursymmetrische Verbindungen bezeichnet werden. Am einfachsten ist es, wenn so, wio in den klassischen Untersuchungen von E. Fischer über die Aldosen, an den Ketten der asymmetrischen Kohlenstoffatome nur ein oder zwei Substituenten umzuwandeln sind.

Unter Benutzung der eben erläuterten Grundsätze lässt sich die Art und Weise, wie die Konfiguration auf Grund der genetischen Beziehungen der untersuchten Verbindungen ermittelt wird, allgemein etwa so darstellen.

Wir gehen aus von der rechtsdrehenden Verbindung $V_{d}^{\prime \prime}$ mit zwei asymmetrischen Kohlenstoffatomen. Bei dem weiteren Ausbau der Verbindung bis auf drei asymmetrische $C$-Atome müssen im allgemeinen Fall, wenn nicht gerade zufällig eine Konfiguration ganz besonders begünstigt ist, nebeneinander $\mathrm{z}$ wei strukturgleiche, stereoisomere Verbindungen entstehen, deren Symbole $V_{x}^{\prime \prime \prime}$ und $V_{y}^{\prime \prime \prime}$ sein sollen. Durch weiteren Ausbau orgiebt sich folgender Stammbaum, in dem übrigens die oberen Indices, die die Anzahl der asymmetrischen Kohlenstoffatome angeben, zur Kennzeichnung nicht unbedingt erforderlich sind.

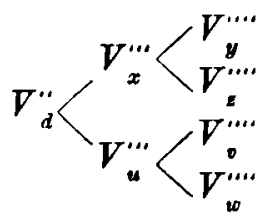

Wir wollen jetzt den graphischen Ort für die Gattungen der Konfiguration dieser Verbindungen aufsuchen. Durch ein dem Stammbaum entsprechendes, in seinen Teilen drehbares Modell lässt sich das folgende ganz besonders anschaulich machen. Für die Bezeichnung der Orte sind in leicht ersichtlicher Weise grosse und kleine Buchstaben verwandt. 
Der Verbindung $\nabla_{d}^{\prime \prime}$ müssen wir wegen der Rechtsdrehung ihres struktursymmetrischen Abkömmlings den Ort $B$ anweisen. Für $V_{x}$ und

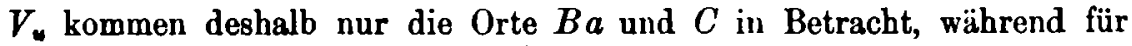
die Verbindungen $V_{y}, V_{z}, V_{v}$ und $V_{w}$ die Orte $B b, C a$ und $D$ in Frage stehen. Aus dem graphischen Felde ergeben sich für diese Orte folgende Konfigurationsgattungen:

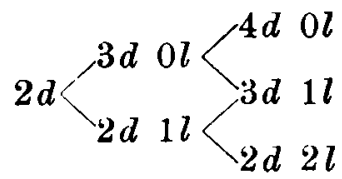

Es muss also eine von den vier Verbindungen $V^{\prime \prime \prime \prime}$ einen Abkömmling liefern, der durch intramolekulare Kompensation in nicht spaltbarer Weise inaktiv ist.

Angenommen, $V_{w}$ liefere diese Verbindung, dann gehört erstere dem Orte $B b$ des graphischen Feldes an. Dann ist aber auch der Ort für $V_{x}$ und $V_{u}$ bestimmt. $V_{x}$ gehört zu $C$, und $V_{u}$ zu $B a$. Da in der Gattung $3 d 0 l$, deren Ort $C$ ist, nur eine Spezies möglich ist, so ist die Konfiguration der Verbindung $V_{x}$ schon allein auf diesem Wege eindeutig bestimmt. Wir erhalten die Konfiguration $d d d$. Da aber aus der nächst niederen Konfiguration $d d$, wenn der Ausbau zur Asymmetrie am Kohlenstoffskelett der Reihe nach fortschreitet, nur die beiden Konfigurationen $d d d$ und $d d l$ entstehen können, so bleibt für die neben $V_{x}$ entstehende strukturgleiche Verbindung $V_{u}$ nur die Konfiguration $d d l$ übrig.

Durch eine Abweichung von der obigen Ausbauregel wird übrigens das Verfahren der Konfigurationsbestimmung nicht wesentlich geändert Die aus der Verbindung $V_{u}$ entstehenden Verbindungen $V_{v}$ und $V_{v}$ können auf Grund der bisherigen Ermittlungen nur das Gefüge $d d l d$ oder ddll haben. $\mathrm{Da}$ sich aber allein der struktursymmetrische $\mathrm{Ab}$ kömmling von $V_{w}$ als nicht spaltbar inaktiv erwiesen hatte, so muss die Konfiguration $d d l l$ der Verbindung $V_{w}$ und die übrigbleibende Konfiguration $d d l d$ der Verbindung $V_{v}$ zukommen. Es ist noch zu entscheiden, welche der beiden Verbindungen $V_{y}$ und $V_{x}$ die Konfiguration $d d d l$ hat. $\mathrm{Zu}$ derselben Gattung gehört die Konfiguration $l d d d$. Die struktursymmetrischen Abkömmlinge von Verbindungen mit diesen beiden Konfigurationen sind jedoch identisch. Bei der Konfiguration $d d d d$ ist eine derartige Erscheinung nicht möglich. Dies giebt uns das Mittel für die Entscheidung an die Hand.

Wenn es sich nur um die Konfigurationsbestimmung einer einzigen 
Verbindung handelt, so ist die Kenntnis des ganzen Stammbaums nicht nötig. So könnte z. B. die weitere Untersuchung der Abkömmlinge von $V^{\prime \prime}$ unterbleiben. Es würde ausreichen nachzuweisen, dass dio struktursymmetrischen Abkömmlinge der aus $V_{x}$ entstehenden Verbindungen $V_{y}$ und $V_{s}$ beide aktiv sind, um dann auf dem obigen Wege etwa die Konfiguration von $\nabla_{z}$ festzustellen.

Man kann schliesslich auch für die Konfigurationsbestimmung (etwa von $V_{s}$ ) auf alle genetischen Beziehungen zu Verbindungen mit der nächst niederen Konfiguration verzichten und die Auswahl unter den (16) möglichen Kombinationen ausschliesslich auf Erscheinungen bei den struktursymmetrischen Abkömmlingen aufbauen. Ausserdem müssen dann jedoch noch besondere Eigenschaftèn der Kette der asymmetrischen $C$ Atome bekannt sein.

Die angedeutete Methodik der Konfigurationsbestimmungen ist zum Teil nur eine Verallgemeinerung von bereits ausgeführten Konfigurationsermittlungen bestimmter Verbindungen. Ich glaube jedoch, dass die hier versuchte Behandlungsweise trotz einer gewissen grösseren Abstraktion das Verständnis der Konfigurationsbestimmungen erleichtern wird. Ausserdem ist es ja zur Vervollkommnung der Theorie immer von Interesse, ein Problem so allgemein als möglich zu behandeln. 